\title{
Spectral Analysis of Quantum-Dash Lasers: Effect of Inhomogeneous Broadening of the Active-Gain Region
}

\author{
Mohammed Zahed Mustafa Khan, Tien Khee Ng, Member, IEEE, Udo Schwingenschlögl, \\ and Boon S. Ooi, Senior Member, IEEE
}

\begin{abstract}
The effect of the active region inhomogeneity on the spectral characteristics of InAs/InP quantum-dash (Qdash) lasers is examined theoretically by solving the coupled set of carrier-photon rate equations. The inhomogeneity due to dash size or composition fluctuation is included in the model by considering dispersive energy states and characterized by a Gaussian envelope. In addition, the technique incorporates multilongitudinal photon modes and homogeneous broadening of the optical gain. The results predict a red shift in the central lasing wavelength of Qdash lasers on increasing the inhomogeneous broadening either explicitly or implicitly, which supports various experimental observations. The threshold current density and the lasing bandwidth are also found to increase.
\end{abstract}

Index Terms - Inhomogeneous broadening, quantum-dash lasers, rate equation model.

\section{INTRODUCTION}

$\mathbf{L}$ ONG wavelength emission from InAs/InP Qdashes, together with a wide gain spectrum and tunable spectral range, has attracted Qdash devices as a potential candidate in long haul fiber-optic communications and networking [1-3]. These achievements are a result of extensive experimental studies on Qdashes from the last decade by different research groups. At device level, Qdash semiconductor optical amplifiers (SOA) and lasers with superior characteristics are demonstrated [1-2]. Besides, some peculiar observations in InAs/InP Qdash lasers, unlike in quantum dot (Qdot) lasers, have been reported. These include, lasing emission typically from the dash ground states (GS) [1-8], and red shift in the lasing wavelength on increasing the number of stack layers [9] or the laser cavity length [6,9]. These features have been attributed to the quasi delta density of state (DOS) of Qdashes and their high-energy tail. Nonetheless, further investigations

Manuscript received December 7, 2011; revised January 18, 2012; accepted January 29, 2012. Date of publication February 24, 2012; date of current version March 6, 2012. This work was supported in part by a Joint Program between KAUST and the University of Michigan, Ann Arbor, under KAUSTAcademic Excellence Alliance 2010 Grant.

M. Z. M. Khan, T. K. Ng, and B. S. Ooi are with the Photonics Laboratory, King Abdullah University of Science and Technology, Thuwal 23955-6900, Saudi Arabia (e-mail: mohammedzahed.khan@kaust.edu.sa; tienkhee.ng@kaust.edu.sa; boon.ooi@kaust.edu.sa).

U. Schwingenschlögl is with the Division of Physical Sciences and Engineering, King Abdullah University of Science and Technology, Thuwal 23955-6900, Saudi Arabia (e-mail: udo.schwingenschlogl@kaust.edu.sa).

Color versions of one or more of the figures in this paper are available online at http://ieeexplore.ieee.org.

Digital Object Identifier 10.1109/JQE.2012.2188852 are required to understand the reason behind these observations in a more comprehensive manner, in particular, the red shift phenomenon. In this respect, theoretical modeling of Qdash devices would serve as a tool to understand the physics behind these observations and thus forms a vital constituent in further improvements of such devices, as already achieved for Qdot devices [10-11]. Various theoretical techniques for modeling the performance characteristics of Qdashes at material level $[2,12-15]$, and device level [3, 16-20], have been reported in literature. However, not many of these models analyzed the spectral characteristics of InAs/InP Qdash lasers except for $[16,17]$, and very recently by our group $[18,19]$, where we made an effort to explain the red shift of lasing wavelength with increasing cavity length or the number of stacking layers by relating these to inhomogeneous broadening (Qdash size of composition dispersion), and considering a qualitative model.

The objective of this paper is to gain a further comprehensive understanding of the red shift phenomenon in Qdash lasers, which has been observed experimentally [6, 9]. For this purpose, a theoretical model is evaluated which is based on the multimode carrier-photon rate equations and the density matrix formulation. Our theoretical calculations validate that the red shift phenomenon is partly associated to the increase in laser active region inhomogeneity together with the quasi zero dimensional DOS of Qdashes. We confirm this relation first by explicitly increasing the inhomogeneous broadening, and later, implicitly, by varying the laser structure parameters (specifically, the cavity length and the number of stacking layers) and comparing with the experimental results. Overall, a good agreement between the numerical and experimental results are observed. Finally, we explore the inhomogeneity effect on the lasing bandwidth (calculated at full width at half maximum (FWHM)) of the Qdash lasers, as this feature has been exploited in the experimental demonstration of broad band Qdash lasers [21-23]. Our results predict an increase in the threshold current density and lasing bandwidth with increase in the active region inhomogeneity, which supports the experimental results. We make an attempt to explain these observations qualitatively.

\section{Theoretical Model}

The InAs/InP Qdash laser structure explored in our work consists of a $w_{W L}=1 \mathrm{~nm}$ thick wetting layer with cross section dash density of $N_{d h}=1.0 \times 10^{12} \mathrm{~cm}^{-2}$ and an active 
region volume of $V_{A}=1.8 \times 10^{-16} \mathrm{~m}^{3}$. The active region comprises four InAs Qdash stacks with an average height of $h_{D h}=1.5 \mathrm{~nm}$ and width of $w_{D h}=20 \mathrm{~nm}$. The $L=1.0 \mathrm{~mm}$ long and $d=40 \mu \mathrm{m}$ stripe width laser has as-cleaved facets that give a reflection coefficient of $R_{1}=R_{2}=0.3$ and an internal loss of $10 \mathrm{~cm}^{-1}$. More details of the structure can be obtained from [5].

Our model which is applicable to InAs/InP Qdash lasers, is based on solving the multimode photon-carrier rate equations to obtain the steady state photons and carriers in each energy state of the conduction band [10]. In addition, the model incorporates the density matrix formulation of the Qdash gain medium where the quantum wire (Qwire) like nature has a large influence on the gain properties of the laser [17]. We consider a three level energy system which includes the separate confinement heterostructure (SCH), the wetting layer (WL) and the ground state (GS) energy level of the Qdashes. For simplicity, we assume that a single GS energy is available inside the dashes for the electrons and holes. The reservoir of carriers is the $\mathrm{SCH}$ layer that receives the injected carriers. Subsequently, these carriers relax to the WL and finally to the GS of the Qdash. The associated time constants are $\tau_{S W}$ (relaxation from $\mathrm{SCH}$ to $\mathrm{WL}$ ), $\tau_{W D}$ (relaxation from $\mathrm{WL}$ to dash GS), $\tau_{D W}$ (re-excitation from dash GS to WL), $\tau_{W S}$ (reexcitation from WL to $\mathrm{SCH}$ ), and $\tau_{S}, \tau_{W}, \tau_{D}$ (recombination in the $\mathrm{SCH}, \mathrm{WL}$ and dash GS layers, respectively). In addition, the model follows similar assumptions as reported in [10]. We group the dashes into $j=0,1, \ldots, 2 M_{d}$ groups according to the interband transition energy and consider $N$ intra-dash energy levels in each dash group characterized by the DOS function $N_{D}$ [17]. Therefore, $E_{j, k}=E_{c v}-\left(M_{d}-j\right) \Delta E_{j}+$ $k \Delta E_{k}$ represents a generic energy level of the system with $E_{j, 0}$ and $E_{j, N}$ being the lowest and highest ground state energies of the $j^{\text {th }}$ dash group, and $j=M_{d}$ corresponds to the central transition energy $E_{c v}$ dash group. In addition, a series of longitudinal photon modes $E_{m}=E_{c v p}-\left(M_{p}-m\right) \Delta E_{m}$ $\left(m=0,1, \ldots, 2 M_{p}\right)$ with central mode energy $E_{c v p}$ and mode separation $\Delta E_{m}=c h / 2 n_{a} L$ is considered $\left(M_{p}\right.$ and $E_{c v p}$ need not be identical to $M_{d}$ and $E_{c v}$, respectively) to describe the interaction between the dashes with different resonant energies through photons.

Based on the density matrix formulation, the linear optical gain of the Qdash active region is given by $[4,10,12]$

$$
g_{m}^{j, k}=\frac{2 \pi e^{2} \hbar N_{D}}{c n_{a} \epsilon_{0} m_{0}^{2}} \frac{\left|M_{c v}\right|^{2}}{E_{c v}}\left(2 P_{j, k}-1\right) G_{j, k} B\left(E_{m}-E_{j, k}\right)
$$

where

$$
N_{D}=N_{d h} \sqrt{2 m_{e}^{*} / \pi^{2} \hbar^{2}} \sqrt{E_{j, N+1}-E_{j, 0}}
$$

is the volumetric DOS with $N_{d h}$ being the cross section dash density; $\left|M_{c v}\right|^{2}$ is the transition matrix, and $P_{j, k}=$ $N_{j, k} / 2 D_{g} N_{D} V_{A} G_{j, k}$ is the carrier occupational probability (including the spin) of the $E_{j, k}^{\text {th }}$ dash group with $N_{j, k}$ carrier population [17].

The optical gain of Eq. 1 which the $E_{j, k}^{t h}$ dash group give to the $m^{\text {th }}$ mode photons, takes into account the Lorentzian homogeneous broadening term

$$
B\left(E_{m}-E_{j, k}\right)=\frac{\hbar \Gamma_{h o m} / 2 \pi}{\left(E_{m}-E_{j, k}\right)^{2}+\left(\hbar \Gamma_{h o m} / 2\right)^{2}}
$$

with FWHM of $\hbar \Gamma_{h o m}$.

$$
\begin{aligned}
G_{j, k}= & \frac{1}{\sqrt{2 \pi} \xi_{0}} \exp \left(\frac{\left(E_{j, 0}-E_{c v}\right)^{2}}{2 \xi_{0}^{2}}\right) d E_{j} \\
& \times \frac{\sqrt{E_{j, k+1}-E_{j, 0}}-\sqrt{E_{j, k}-E_{j, 0}}}{\sqrt{E_{j, N+1}-E_{j, 0}}}
\end{aligned}
$$

is the fraction of energy states available at the $E_{j, k}$ energy level. The first term on the right hand side of Eq. 4 correspond to the Gaussian inhomogeneous broadening (GIB) due to dash size fluctuation, particularly the height, with FWHM of $\Gamma_{i n h}=$ $2.35 \xi_{0}\left(\xi_{0}\right.$ being the standard deviation), while the second term is the ratio of two integrals [17]. Note that we have normalized $G_{j, k}$ as $\sum_{j, k} G_{j, k}=1$. The rate equations are as follows:

$$
\begin{aligned}
\frac{d N_{S}}{d t}= & \frac{\eta_{i} I}{e}-\frac{N_{S}}{\tau_{S W}}-\frac{N_{S}}{\tau_{S}}+\frac{N_{W}}{\tau_{W S}} \\
\frac{d N_{W}}{d t}= & \frac{N_{S}}{\tau_{S W}}+\sum_{j} \sum_{k} \frac{N_{j, k}}{\tau_{D W}^{j, k}}-\frac{N_{W}}{\overline{\tau_{W D}}}-\frac{N_{W}}{\tau_{W S}}-\frac{N_{W}}{\tau_{W}} \\
\frac{d N_{j, k}}{d t}= & \frac{N_{W} G_{j, k}}{\tau_{W D}^{j, k}}-\frac{N_{j, k}}{\tau_{D W}^{j, k}}-\frac{N_{j, k}}{\tau_{D}}-\frac{c \Gamma}{n_{a}} \sum_{m} g_{m}^{j, k} S_{m} \\
\frac{d S_{m}}{d t}= & \beta \sum_{k} \sum_{j} B\left(E_{m}-E_{j, k}\right) \frac{N_{j, k}}{\tau_{S p}} \\
& +\frac{c \Gamma}{n_{a}} \sum_{k} \sum_{j} g_{m}^{j, k} S_{m}-\frac{S_{m}}{\tau_{p}} .
\end{aligned}
$$

The first three rate equations refer to the carrier dynamics in the $\mathrm{SCH}, \mathrm{WL}$ and dash GS energy levels with carrier populations $N_{S}, N_{W}$ and $N_{j, k}$, respectively. $I$ is the current injection, $\eta_{i}$ is the internal quantum efficiency, $\overline{\tau_{W D}}$ (average carrier relaxation lifetime from WL to GS) and $\tau_{W D}^{j, k}$ are calculated according to: $[10,17]$

$$
\begin{aligned}
\left(\overline{\tau_{W D}}\right)^{-1} & =\sum_{j} \sum_{k}\left(\tau_{W D}^{j, k}\right)^{-1} G_{j, k} \\
& =\sum_{j} \sum_{k} \tau_{W D_{0}}^{-1}\left(1-P_{j, k}\right) G_{j, k} .
\end{aligned}
$$

$\tau_{S p}$ is the spontaneous emission life time and the photon lifetime is calculated as: [10]

$$
\tau_{p}^{-1}=\left(\frac{c}{n_{a}}\right)\left(\alpha_{i}+\frac{\ln \left(1 / R_{1} R_{2}\right)}{2 L}\right)
$$

where $n_{a}=3.5$ correspond to the refractive index of the active region and $c$ is the velocity of light in vacuum. $\tau_{D W}$ is calculated through the condition of detailed balance as: [17]

$$
\begin{aligned}
\left(\tau_{D W}^{j, k}\right)^{-1} & =\left(1-\frac{N_{W}}{2 D_{W} V_{W}}\right)\left(\tau_{D W_{0}}^{j, k}\right)^{-1} \\
\tau_{D W_{0}}^{j, k} & =\left(\frac{D_{G} N_{D} V_{A}}{D_{W} V_{W}}\right) \tau_{W D_{0}} \exp \left(\frac{E_{W L}-E_{j, k}}{k T}\right) .
\end{aligned}
$$


$D_{G}, D_{W}$ and $E_{W L}$ represent the GS degeneracy of the Qdash, the volumetric DOS of WL and the WL energy level, respectively. $V_{A}=0.75 h_{D h} d L N_{l y r}$ and $V_{W}=w_{W L} d L N_{l y r}$, corresponds to the volume of the active region and WL, respectively, where $N_{l y r}$ is the number of stacking layers. Eq. 8 corresponds to the multimode photon dynamics with photon population $S_{m}$, where the second and third terms on the right hand side represent the stimulated emission and photon loss, respectively.

The steady state carrier and the photon population are obtained by solving the rate equations (Eqns. 5-8) with the fourth-order Runge-Kutta numerical method by applying an input step current at $t=0$. Subsequently, the laser output power of the $m^{t h}$ mode photon of energy $\hbar \omega_{m}$ from one cavity facet is obtained by [10]:

$$
I_{m}=\hbar \omega_{m} c S_{m} \ln (1 / R) /\left(2 L n_{a}\right) .
$$

\section{RESULTS AND DISCUSSION}

The parameters used in the model are as follows: [10, 17] $E_{c v}=805 \mathrm{meV}, E_{W L}=916 \mathrm{meV}, \hbar \Gamma_{h o m}=10 \mathrm{meV}$, optical confinement factor $\Gamma=0.03$, spontaneous emission efficiency and lifetime $\beta=10^{-4}$ and $\tau_{S p}=2.8 \mathrm{~ns}$. The recombination lifetimes within the $\mathrm{SCH}, \mathrm{WL}$ and Qdash GS are, respectively, $\tau_{S}=\infty, \tau_{W}=0.8 n s$ and $\tau_{D}=0.5 n s$, while the carrier capture and escape in the SCH are $\tau_{S W}=$ $0.5 n s$ and $\tau_{W S}=1.0 n s$, respectively.

The degeneracy of the WL and GS is taken as $D_{W}=1.8 \times$ $10^{19} \mathrm{~cm}^{-3}$ and $D_{G}=1$, respectively, and the volumetric DOS is $N_{D}=5 \times 10^{17} \mathrm{~cm}^{-3}$ [17]. The initial carrier relaxation lifetime from WL to dash GS is taken as $\tau_{W D 0}=2 p s$, while the separation between the dash groups is $\Delta E_{j}=0.354 \mathrm{meV}$ and $M_{d}$ (100 to 701) varies according to the inhomogeneous broadening value (15 to $85 \mathrm{meV}$ ). These are the converged values of the dash groups once stabilizing the output power and lasing spectra in each case.

\section{A. Explicit Increase in Inhomogeneous Broadening}

Fig. 1(a) shows the room temperature calculated results of the threshold central lasing wavelength $\left(\lambda_{c, t h}\right)$, calculated by identifying the central wavelength at the FWHM, and the threshold current density $\left(J_{t h}\right)$ as a function of active region inhomogeneity $\left(\Gamma_{i n h}\right)$ which is varied explicitly by fixing entire laser structure parameters. A red shift in $\lambda_{c, t h}$ and a substantial increase in $J_{t h}$ is observed on increasing the inhomogeneous broadening. The threshold current density approximately doubles in value and the central threshold wavelength shifts $\sim 20 \mathrm{~nm}$ towards the longer wavelengths when $\Gamma_{i n h}$ is increased by five times. This observation has been partly attributed to the fluctuation in the size of the Qdashes, particularly the height, and its DOS, by considering a qualitative model [18]. In the following we make an effort to explain the observation from a different viewpoint qualitatively. In the case of the smaller inhomogeneous broadening, the dash sizes would preferably be similar due to their comparable heights. Therefore, population inversion might occur in most of the Qdashes simultaneously at low current injection owing to their
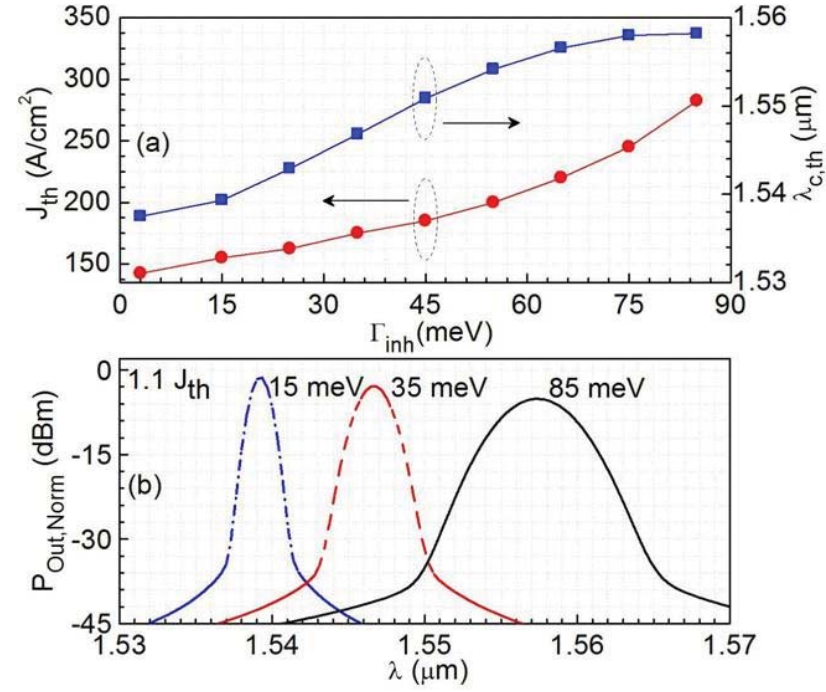

Fig. 1. Calculated (a) threshold current density and central threshold wavelength at various values of Qdash inhomogeneity and (b) lasing spectra at low injection current $\left(1.1 J_{\text {th }}\right)$ for three different inhomogeneous broadening values, corresponding to the Qdash laser reported in [5]. P Put,Norm is the calculated normalized output power.

moderately confined structures and similar band transition energies. However, Qdash assembly with dispersive sizes (fluctuating heights) would not satisfy the lasing conditions at the same low current injection. Dashes with sizes that are comparable to the electron wavelength acquire dot-like features with relatively tight confinement in all directions. Hence, lower modal gain and DOS of these small dashes require a smaller number of carriers to achieve lasing conditions. The generated high-energy photons from these small dashes would then be absorbed by large dashes with shorter band transition energies because of their comparatively large height. To facilitate lasing from the highly inhomogeneous Qdash laser structure, high injection of carriers is necessary to populate sufficient carriers in large dashes, and drive them to lase. Therefore, larger dashes prominently dominate the lasing condition of the structure due to their higher modal gain and DOS, in general. Because of their smaller band transition energy, lasing occurs in the longer wavelength region, thus contributing to a red shift in the central lasing wavelength.

Fig. 1(b) shows the calculated lasing spectra above threshold $\left(1.1 J_{t h}\right)$ at three different inhomogeneous broadening values. Note that the lasing bandwidth $(\Delta \lambda)$ increases by four times, attaining a value of $\sim 4.5 \mathrm{~nm}$ at $\Gamma_{i n h}=85 \mathrm{meV}$, compared to $\sim 1.1 \mathrm{~nm}$ at $\Gamma_{i n h}=15 \mathrm{meV}$. On the other hand, the peak output power decreases and the total output power increases for a large $\Gamma_{i n h}$ system. This observation is consistent with recent experimental studies on the broadband Qdash laser [21-23]. This might be due to an insufficient lower energy DOS for the carriers to occupy in the case of a highly inhomogeneous active region. Hence, they move to the highenergy region of the DOS, thereby enhancing the lasing bandwidth even at lower current injection. Moreover, the large dispersion in dash sizes essentially isolate various dash groups. Thus, each group starts lasing independently and con- 

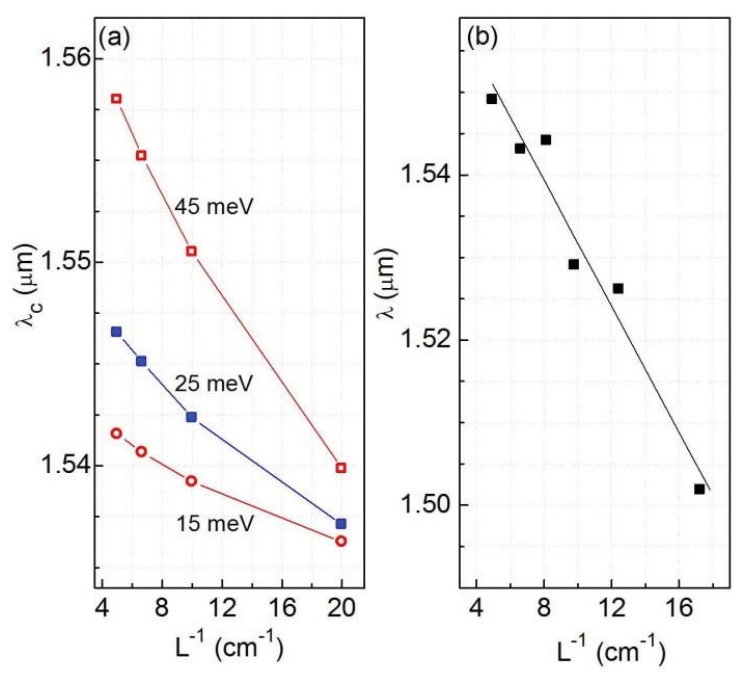

Fig. 2. (a) Calculated central lasing wavelength at different cavity lengths for low $\left(1.1 J_{\text {th }}\right)$ injection current. (b) Experimental results of the lasing wavelength at different cavity lengths of a similar laser structure [9]. The numbers in (a) correspond to the inhomogeneous broadening value.

currently because they are widely separated from the central lasing mode. As a consequence, less peak optical power can be emitted under the same current injection and with enhanced FWHM of the lasing spectra.

\section{B. Comparison With the Experimental Results}

In the above section, we have discussed the dependence of the lasing wavelength on the active medium inhomogeneity by varying $\Gamma_{i n h}$ in Eq. 4 explicitly by fixing the entire laser parameters. In practice, we show how these results might be related to various experimental studies on InAs/InP Qdash lasers reported in literature [6, 8-9]. In general, the active region inhomogeneity also depends inherently on the laser structure parameters such as the cavity length and the number of stacking layers. Therefore, we consider their dependence on the lasing wavelength by fixing $\Gamma_{i n h}$ and varying these parameters instead. Such an alteration of $\Gamma_{i n h}$ due to the laser parameters is termed as implicit increase in inhomogeneous broadening. Increasing the cavity length increases the planar area of the laser active region (and hence the volume) and subsequently incorporate relatively more dashes as compared to the smaller active region volume (shorter cavity with small planar area) laser. Hence, the probability of size dispersion might be higher in the longer cavity laser than the shorter cavity laser. Similarly, increasing the number of stacking layers increases the active region volume by including a larger density of dashes with larger size fluctuation thus, enhancing the active region inhomogeneity.

Fig. 2(a) shows the calculated results of the central lasing wavelength $\left(\lambda_{c}\right)$ as a function of cavity length, and at various inhomogeneous broadening values, for the Qdash laser reported in [5]. The experimental study of this effect on a similar InAs/InP Qdash laser [9] is plotted in Fig. 2(b) which depict that increasing the cavity length red shift the lasing wavelength. This phenomenon is well reproduced by our model at different $\Gamma_{i n h}$ values. We attribute this red

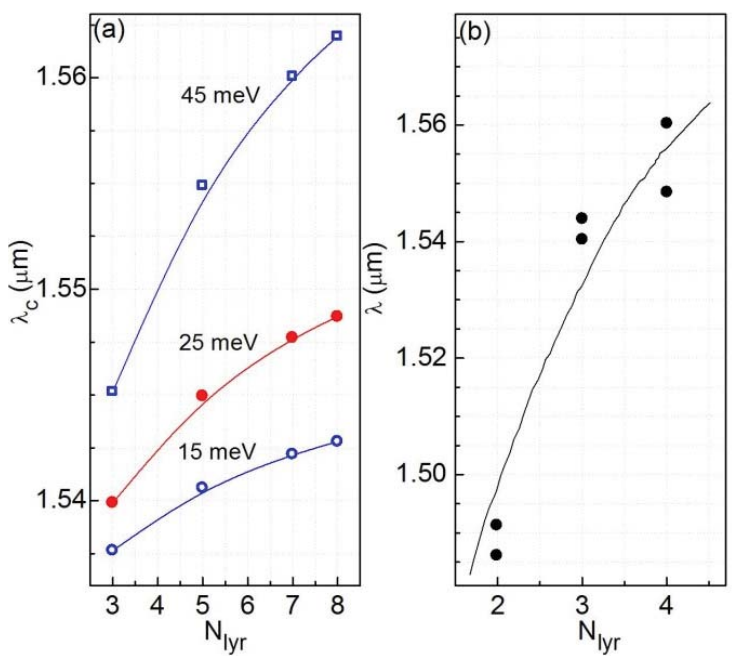

Fig. 3. (a) Calculated central lasing wavelength at different stacking layers for low $\left(1.1 J_{\text {th }}\right)$ injection current. (b) Experimental results of the lasing wavelength at different number of stacking layers of a similar laser structure [9]. The numbers in the inset of (a) correspond to the inhomogeneous broadening value.

shift phenomenon partly to the increase in $J_{t h}$ as the cavity length decreases which might blue shifts the lasing wavelength and thereby providing an impression of red shift phenomenon, as was the case with quantum well lasers [24, 25]. In addition, this observation may partly be ascribed to the implicit increase in inhomogeneous broadening, as the cavity length increases, thus red shifting the lasing wavelength. In order to demonstrate this possible attribution, we utilized an indirect approach of plotting the red shift phenomenon at different $\Gamma_{i n h}$ values, as shown in Fig. 2(a). We observed that increasing the inhomogeneous broadening enhanced the red shift phenomenon substantially. For a smaller inhomogeneous broadening $\left(\Gamma_{i n h}=15 \mathrm{meV}\right)$ the total red shift constitutes $\sim 5.3 \mathrm{~nm}$ when the cavity length is varied from 0.5 to $2.0 \mathrm{~mm}$ while the experimental results show $\sim 47 \mathrm{~nm}$. However, for the case of $\Gamma_{i n h}=45 \mathrm{meV}$, the red shift phenomenon is $\sim 18.5 \mathrm{~nm}$ which is more than three times that of the smaller inhomogeneous broadening. Therefore, the enhancement in the total red shift validates that the red shift phenomenon is partly attributed to the increase in $\Gamma_{i n h}$ implicitly incorporating the unique DOS of Qdashes, and perhaps seems to be a dominating parameter. Moreover, the above conclusion also supports the recent experimental studies of the cavity effect on the lasing spectra of InAs/InP lasers [6], where the authors attribute the red shift phenomenon to the DOS of the Qdashes. As a final point, the above statement is substantiated by the fact that the model does not consider any growth/processing parameters that affects the lasing wavelength.

Note that the lasing wavelength at various cavity lengths is relatively different in Figs. 2(a) and (b). This is anticipated since the two laser structures are different in nature and therefore possess different structural parameters. Moreover, we utilized a staircase approximation to model the energy states of Qdashes according to Eqs. 2 and 4. Our aim in this work is to investigate the spectral characteristics of Qdash lasers and not to model the energy states of the dashes accurately. 
The red shift phenomenon has also been studied experimentally by varying the number of stacking layers $\left(N_{l y r}\right)$ on the lasing wavelength by Zhou et al. [9]. The authors observed a substantial increase in the lasing wavelength $(\sim 67 \mathrm{~nm})$ on increasing the stack number from 2 to 4 , which, they have attributed to the increase in inhomogeneous broadening and the DOS of Qdashes. In order to investigate this observation we simulated the InAs/InP laser structure [5] at different stack number by including appropriate confinement factor values for each stack number, and are presented in Fig. 3. The other parameters are unaltered. The experimental data from [9] is also plotted in Fig. 3(b) for comparison purpose. Our simulation results do show a red shift in the central lasing wavelength as a function of stack number and at fixed active region inhomogeneity. This observation might be attributed to the decrease in the optical confinement factor as the number of stacking layers increases which is comprehensively studied by our group recently [19]. In addition, Fig. 3(a) show that the red shift phenomenon enhanced with increase in inhomogeneous broadening of the active region. We observed a total red shift of $\sim 5.2 \mathrm{~nm} f$ or the smaller inhomogeneous broadening $\left(\Gamma_{i n h}=15 \mathrm{meV}\right)$ and $\sim 17 \mathrm{~nm}$ for $\Gamma_{i n h}=45 \mathrm{meV}$. An enhancement by more than three times is obtained when compared to the smaller inhomogeneous broadening. Thus, in this case also, we ascribe the red shift phenomenon partly to the implicit increase in active region inhomogeneity which is demonstrated above in an indirect mode.

In general, our simulation results are in good agreement with the experimental observation as depicted in Figs. 2 and 3 thus showing the effectiveness of our model. However, as mentioned earlier, the lasing wavelengths of the simulation results and does not agree with the experiment owing to the fact that the laser structures are different. Moreover, the observed red shift phenomenon, in either of the two cases, could be realized either by our qualitative explanation that we provided in the previous section from a different viewpoint, or by our qualitative model [18] wherein we explained this phenomenon relating fairly to the DOS of Qdashes.

Therefore, in view of above discussion, we can now generalize that the central lasing wavelength depends on either explicit increase or implicit increase (as a result of increase in the number of stacking layers or the cavity length or both) of active region inhomogeneity, and deduce a relation for the central lasing wavelength based on these observations as $\lambda_{c} \propto \Gamma_{i n h} L N_{l y r}$.

\section{Conditions to Achieve Red Shift Phenomenon}

We have seen in the previous sections that the inhomogeneous broadening is possibly the dominating candidate for the observation of red shift phenomenon which we have demonstrated through our rate equation model. However, care must be exercised in selecting proper parameter values because, through a number of simulations, our model revealed occurrence of critical parameters for this observation. These are the total loss of the laser structure $\alpha=\alpha_{i}+\ln \left(1 / R_{1} R_{2}\right) / 2 L$ that includes the cavity loss, and the volumetric DOS value $N_{D}$. In order to understand the influence of these parameters
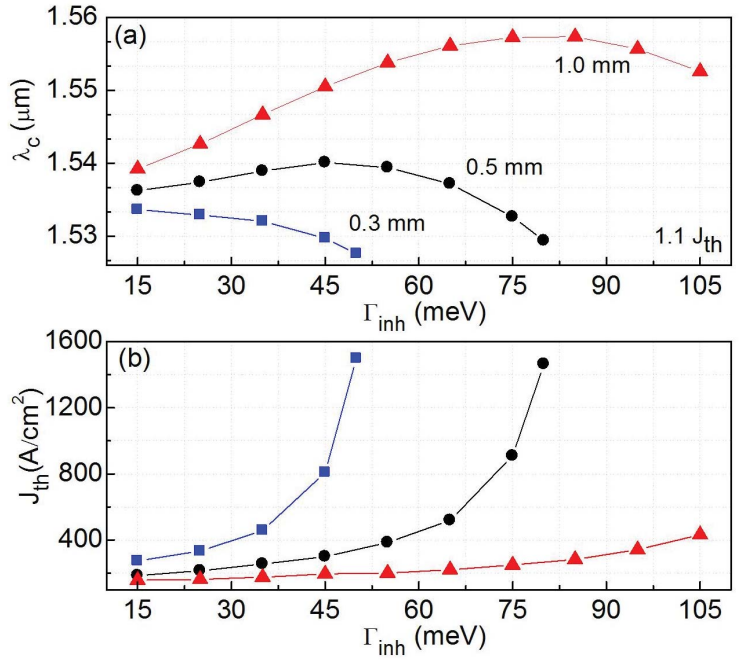

Fig. 4. Calculated (a) central lasing wavelength and (b) threshold current density at different cavity lengths, as a function of active region inhomogeneity, and at low $\left(1.1 J_{\text {th }}\right)$ injection current.

comprehensively, we show the effect of $\lambda_{c}$ as a function of $\Gamma_{i n h}$, and at different cavity lengths, in Fig. 4(a). The remaining laser parameters are unaltered (including $N_{D}$ ). Note that, for the case of $1.0 \mathrm{~mm}$ and $0.5 \mathrm{~mm}$ cavity lengths, increasing $\Gamma_{i n h}$ first shifts $\lambda_{c}$ to longer wavelengths and later, to shorter wavelengths. However, for the case of $0.3 \mathrm{~mm}$ cavity length, no red shift phenomenon is observed and instead increasing $\Gamma_{i n h}$ shifts $\lambda_{c}$ to shorter wavelengths. In order to understand this unusual observation, we relate this to the total loss of the laser which decreases with increase in cavity length. The calculated $\alpha$ for $1.0,0.5$, and $0.3 \mathrm{~mm}$ cavity lengths are 22, 34, and $50 \mathrm{~cm}^{-1}$, respectively. Considering the longer cavity laser, the selected value of $N_{D}$ might be large enough to compensate for the total loss at the inhomogeneous broadening values of $\Gamma_{i n h} \leq 85 \mathrm{meV}$ and thus showing the red shift phenomenon. In other words, the weighted DOS (the fraction of the energy states available from the total $N_{D}$ energy states) of the larger dashes were enough to compensate for the total loss when $\Gamma_{i n h}$ increases from $15 \mathrm{meV}$ to $\leq 85 \mathrm{meV}$, and hence their dominating nature, when compared to smaller dashes, persists. Note that the weighted DOS value decreases (flattens) with increase in $\Gamma_{i n h}$ (Eqns. 2 and 4), yet, we observe the red shift phenomenon till $\Gamma_{\text {inh }} \leq 85 \mathrm{meV}$ probably due to the availability of just enough energy states to compensate for the total loss. However, when the laser active region inhomogeneity is increased beyond $85 \mathrm{meV}$, the available energy states in the larger dashes might not be enough to reach threshold and support lasing as a result of decrease in their weighted DOS value (deficiency in the DOS), and thus, losing their dominating nature. Now, the smaller dashes come into picture and lasing occurs via them at shorter wavelengths since they reach threshold earlier than the larger dashes because of their dot-like features. Therefore, the total loss is now compensated collectively from larger and smaller dashes with smaller dashes becoming dominant. This probably is the reason for the observation of blue shift in the lasing wavelength when $\Gamma_{i n h}>85 \mathrm{meV}$. We define 
TABLE I

EFfect of Volumetric Density of States on the Transition INHOMOGENEOUS BROADENING AT $1.1 J_{\text {th }}$

\begin{tabular}{c|c}
\hline \hline$N_{D}\left(\mathrm{~cm}^{-3}\right)$ & $\Gamma_{i n h}^{T}(\mathrm{meV})$ \\
\hline $3 \times 10^{17}$ & 25 \\
\hline $5 \times 10^{17}$ & 45 \\
\hline $7 \times 10^{17}$ & 65 \\
\hline \hline
\end{tabular}

$\Gamma_{i n h}=85 \mathrm{meV}$ as the transition inhomogeneous broadening value $\left(\Gamma_{i n h}^{T}\right)$ below which red shift phenomenon is observed and above which blue shift is observed. Moreover, the above explanation is substantiated by noticing a sharp increase in $J_{t h}$ above $\Gamma_{i n h}^{T}$, as shown in Fig. 4(b). This might be due to substantial increase in the high-energy photon generation by the smaller dashes because part of the photon population gets absorbed in the larger dashes and remaining contribute to the stimulated emission.

In the case of smaller cavity lasers, $\Gamma_{i n h}^{T}$ reduced to $45 \mathrm{meV}$ for $0.5 \mathrm{~mm}$ cavity length, and no $\Gamma_{i n h}^{T}$ is observed for $0.3 \mathrm{~mm}$ cavity laser. This is an expected behavior since $N_{D}$ is fixed for all the cases. For instance, considering the $0.5 \mathrm{~mm}$ cavity laser, more number of energy states (higher weighted DOS) are required in the larger dashes to compensate for the total loss of $34 \mathrm{~cm}^{-1}(\sim 1.5$ times the loss of longer cavity laser) thus leads to the decrease in the $\Gamma_{i n h}^{T}$ value.

Next, we explored the second parameter $\left(N_{D}\right)$ that affects the red shift phenomenon observation. In this case, we fixed the total loss by fixing the cavity length to $0.5 \mathrm{~mm}$ and varying $N_{D}$. The remaining parameters are unaltered. The results are tabulated in Table 1. As expected, the transition inhomogeneous broadening value increased with increase in $N_{D}$ and thus enhances the range of $\Gamma_{i n h}$ for the observation of red shift phenomenon. This probably is due to increase in the weighted DOS (more number of energy states available in the larger dashes) due to increase in $N_{D}$ value which enables larger dashes to maintain its dominance at even larger $\Gamma_{i n h}$ values. It is worth mentioning that increasing $N_{D}$ more than two times has enhanced $\Gamma_{i n h}^{T}$ by more than two and a half times in value. Therefore, a proper selection of these two parameters becomes important for the correct realization of Qdash laser performance when utilizing the presented rate equation model.

\section{Broadening of the Qdash Lasing Spectra}

Finally, we have applied the model to the InAs/InP Qdash laser [5] to investigate the effect of injection current. For this case, we have fixed the cavity length to $0.5 \mathrm{~mm}$ and other parameters remain unaltered. The results of the variation of the central lasing wavelength as a function of active region inhomogeneity is illustrated in Fig. 5(a) for low $\left(1.1 J_{t h}\right)$ and high $\left(5.0 J_{t h}\right)$ current injection values. We have assumed that the homogeneous broadening does not change with the current injection. Apart from showing a red shift with increasing the inhomogeneity of the Qdash gain medium, the figure also reveals a blue shift in the central lasing wavelength at
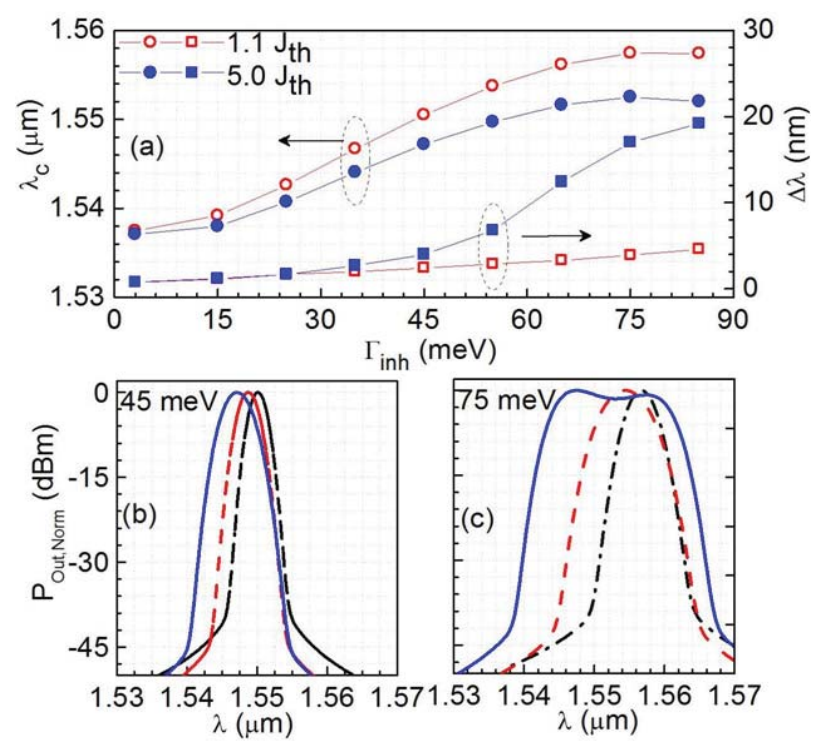

Fig. 5. (a) Calculated lasing bandwidth and central lasing wavelength at different Qdash inhomogeneity values, for low $\left(1.1 J_{\text {th }}\right)$ and high $\left(5.0 J_{\text {th }}\right)$ injection currents of the Qdash laser reported in [5]. (b) and (c) Calculated lasing spectra at $1.5 J_{\text {th }}$ (dashed-dotted line), $3.0 J_{\text {th }}$ (dashed line), and $5.0 J_{\text {th }}$ (solid line) for $\Gamma_{\text {inh }}=45$ and $75 \mathrm{meV}$, respectively. $P_{\text {Out,Norm }}$ is the calculated normalized output power.

high current injection (above threshold) which enhances with inhomogeneous broadening. More prominently, this aspect can be noticed in Figs. 5(b) and 5(c) where the lasing spectra corresponding to $\Gamma_{i n h}=45 \mathrm{meV}$ and $\Gamma_{i n h}=75 \mathrm{meV}$ are plotted for different current injections. The blue shift is visible in both cases. This may be due to the band filling effect where both the lasing bandwidth and $\lambda_{c}$ move towards higher energy transitions. In general, the carriers fill the lowest GS energy level of the Qdashes from the available wide energy levels as determined by their DOS function (which includes a dominant GS followed by a high energy tail) and contribute to the lasing. With increasing the current injection, more carriers are forced to occupy higher energy levels once the lower energy states are completely filled by carriers that constantly recombine in the system. This results in high energy transitions corresponding to a blue shift of the central lasing wavelength and the lasing bandwidth.

Fig. 5(a) also illustrates the broadening of the lasing spectra at both low and high current injection, on increasing the active medium inhomogeneity. This is generally due to a constant recombination of carriers at lower and higher energy levels in Qdashes. However, the broadening is small in the case of the less inhomogeneous medium even at high current injection (see Fig. 5(b)). This is attributed to the decrease of overlapping energy levels (particularly in the high energy region), and hence DOS, for the electrons to occupy. Thus, the broadening of lasing spectra due to active region inhomogeneity, is minute in this case. But it is worth noticing that under high carrier injection, if the inhomogeneous broadening is increased, the lasing bandwidth increases substantially, as is shown in Figs. 5(a) and 5(c). The lasing bandwidth attains a value of $\sim 4 \mathrm{~nm}$ at $5.0 J_{t h}$ for $\Gamma_{i n h}=45 \mathrm{meV}$. On the contrary, at the same current injection, the Qdash laser with 
$\Gamma_{\text {inh }}=75 \mathrm{meV}$ attains a value of $\sim 18 \mathrm{~nm}$ and exceeds $20 \mathrm{~nm}$ for $\Gamma_{i n h}=85 \mathrm{meV}$, which is approximately five times wider. From the experimental studies on the recently reported broadband Qdash laser [21-23], the lasing bandwidth achieved with $1.0 \mathrm{~mm}$ cavity length is $\sim 50 \mathrm{~nm}$. Therefore, our theoretical results agree with the experimental observation, although the bandwidths achieved are not similar. The increase in the lasing bandwidth is due to the availability of highly dispersive GS energy levels of the Qdashes (due to large fluctuation in size) and nearly complete overlap of their high energy tail states (determined by the DOS). Therefore, a higher DOS in the high energy transition region of the dashes might contribute to broadening of the lasing bandwidth by constant recombination in the system. These combined effects generally broaden the lasing spectra and lead to blue shifts of the lasing bandwidth and $\lambda_{c}$. Another possible explanation would be simultaneous lasing from both smaller and larger dashes, resulting in broadening of the lasing spectra.

\section{CONCLUSion}

In conclusion, we have analyzed the inhomogeneous broadening effect on the characteristics of InAs/InP Qdash lasers explicitly and observed the red shift phenomenon. We have validated the proposal that this phenomenon is partly connected to the quasi zero dimensional DOS of Qdashes together with the active region inhomogeneity, and explained the observation qualitatively from another perspective. We also have analyzed the inhomogeneous broadening effect implicitly by relating it to the laser structure parameters such as cavity length and stack number, observing the red shift phenomenon and comparing it to the experiment. Our model predicts an increase in the threshold current density and lasing bandwidth on increasing the inhomogeneous broadening value.

\section{REFERENCES}

[1] F. Lelarge, B. Dagens, J. Renaudier, R. Brenot, A. Accard, F. van Dijk, D. Make, O. Le Gouezigou, J.-G. Provost, F. Poingt, J. Landreau, O. Drisse, E. Derouin, B. Rousseau, F. Pommereau, and G.-H. Duan, "Recent advances on InAs/InP quantum dash based semiconductor lasers and optical amplifiers operating at $1.55 \mu \mathrm{m}$," IEEE J. Sel. Topics Quantum Electron., vol. 13, no. 1, pp. 111-124, Jan.-Feb. 2007.

[2] J. P. Reithmaier, A. Somers, S. Deubert, R. Schwertberger, W. Kaiser, A. Forchel, M. Calligaro, P. Resneau, O. Parillaud, S. Bansropun, M. Krakowski, R. Alizon, D. Hadass, A. Bilenca, H. Dery, V. Mikhelashvili, G. Eisenstein, M. Gioannini, I. Montrosset, T. W. Berg, M. Poel, J. Mørk, and B. Tromborg, "InP based lasers and optical amplifiers with wire/dot-like active regions," J. Phys. D: Appl. Phys., vol. 38, no. 13, pp. 2088-2102, 2005.

[3] N. A. Naderi, M. Pochet, F. Grillot, N. B. Terry, V. Kovanis, and L. F. Lester, "Modeling the injection-locked behavior of a quantum dash semiconductor laser," IEEE J. Sel. Topics Quantum Electron., vol. 15, no. 3, pp. 563-571, May-Jun. 2009.

[4] Z. Mi and P. Bhattacharya, "DC and dynamic characteristics of P-doped and tunnel injection 1.65- $\mu \mathrm{m}$ InAs quantum-dash lasers grown on InP (001)," IEEE J. Quantum Electron., vol. 42, no. 12, pp. 1224-1232, Nov. 2006.

[5] R. Schwertberger, D. Gold, J. P. Reithmaier, and A. Forchel, "Longwavelength InP-based quantum-dash lasers," IEEE Photon. Technol. Lett., vol. 14, no. 6, pp. 735-737, Jun. 2002.

[6] C. L. Tan, H. S. Djie, Y. Wang, C. E. Dimas, V. Hongpinyo, Y. H. Ding, and B. S. Ooi, "The influence of nonequilibrium distribution on room-temperature lasing spectra in quantum-dash lasers," IEEE Photon. Technol. Lett., vol. 21, no. 1, pp. 30-32, Jan. 2009.
[7] R. Wang, A. Stintz, P. M. Varangis, T. C. Newell, H. Li, K. J. Malloy, and L. F. Lester, "Room-temperature operation of InAs quantum-dash lasers on InP (001)," IEEE Photon. Technol. Lett., vol. 13, no. 8, pp. 767-769, Aug. 2002.

[8] D. Zhou, R. Piron, F. Grillot, O. Dehaese, E. Homeyer, M. Dontabactouny, T. Batte, K. Tavernier, J. Even, and S. Loualiche, "Study of the characteristics of $1.55 \mu \mathrm{m}$ quantum dash/dot semiconductor lasers on InP substrate," Appl. Phys. Lett., vol. 93, no. 16, pp. 161104-1-1611043, 2008.

[9] D. Zhou, R. Piron, M. Dontabactouny, E. Homeyer, O. Dehaese, T. Batte, M. Gicquel, F. Grillot, K. Tavernier, J. Even, and S. Loualiche, "Effect of stack number on the threshold current density and emission wavelength in quantum dash/dot lasers," Phys. Status Solidi (c), vol. 6, no. 10, pp. 2217-2221, 2009

[10] M. Sugawara, K. Mukai, Y. Nakata, and H. Ishikawa, "Effect of homogeneous broadening of optical gain on lasing spectra in self-assembled $\mathrm{In}_{x} \mathrm{Ga}_{1-x} \mathrm{As} / \mathrm{GaAs}$ quantum dot lasers," Phys. Rev. B, vol. 61, no. 11, pp. 7595-7603, 2000.

[11] C. Tan, Y. Wang, H. S. Djie, and B. S. Ooi, "Role of optical gain broadening in the broadband semiconductor quantum-dot laser," Appl. Phys. Lett., vol. 91, no. 6, pp. 061117-1-061117-3, 2009.

[12] M. Gioannini, "Numerical modeling of the emission characteristics of semiconductor quantum dash materials for lasers and optical amplifiers," IEEE J. Quantum Electron., vol. 40, no. 4, pp. 364-373, Apr. 2004.

[13] M. Gioannini, "Investigation of p-type doping effect on the gain characteristics of quantum dash semiconductor lasers," Proc. SPIE, vol. 5452, pp. 527-534, Sep. 2004.

[14] O. Qasaimeh, "Differential gain of closely spaced energy states in quantum dashes," J. Lightw. Technol., vol. 28, no. 13, pp. 1906-1912, Jul. 2010.

[15] J. Wei and K. Chan, "A theoretical analysis of quantum dash structures," J. Appl. Phys., vol. 97, no. 12, pp. 123524-1-123524-12, 2005.

[16] H. Dery and G. Eisenstein, "Self-consistent rate equations of selfassembly quantum wire lasers," IEEE J. Quantum Electron., vol. 40, no. 10, pp. $1398-1409$, Oct. 2004.

[17] D. Hadass, A. Bilenca, R. Alizon, H. Dery, V. Mikhelashvili, G. Eisenstein, R. Schwertberger, A. Somers, J. P. Reithmaier, A. Forchel, M. Calligaro, S. Bansropun, and M. Krakowski, "Gain and noise saturation of wide-band InAs-InP quantum dash optical amplifiers: Model and experiments," IEEE J. Sel. Topics Quantum Electron., vol. 11, no. 5, pp. 1015-1026, Sep.-Oct. 2005.

[18] M. Z. M. Khan, T. K. Ng, U. Schwingenschlogl, P. Bhattacharya, and B. S. Ooi, "Modeling the lasing spectra of InAs/InP quantum dash lasers," Appl. Phys. Lett., vol. 98, no. 10, pp. 101105-1-101105-3, 2011.

[19] M. Z. M. Khan, T. K. Ng, U. Schwingenschlogl, P. Bhattacharya, and B. S. Ooi, "Effect of the number of stacking layers on the characteristics of quantum-dash lasers," Opt. Exp., vol. 19, no. 14, pp. 13378-13385, 2011.

[20] O. Qasaimeh, "Low-current N-type quantum dash semiconductor optical amplifiers," IEEE Photon. Technol. Lett., vol. 21, no. 19, pp. 1390-1392, Oct. 2009.

[21] H. Djie, C. L. Tan, B. S. Ooi, J. C. M. Hwang, X.-M. Fang, Y. Wu, J. M. Fastenau, W. K. Liu, G. T. Dang, and W. H. Chang, "Ultrabroad stimulated emission from quantum-dash laser," Appl. Phys. Lett., vol. 91, no. 11, pp. 111116-1-111116-3, 2007.

[22] B. S. Ooi, H. S. Djie, Y. Wang, C.-L. Tan, J. C. M. Hwang, X.-M. Fang, J. M. Fastenau, A. W. K. Liu, G. T. Dang, and W. H. Chang, "Quantum dashes on InP substrate for broadband emitter applications," IEEE J. Sel. Topics Quantum Electron., vol. 14, no. 4, pp. 1230-1238, Jul.-Aug. 2008.

[23] C. Tan, H. S. Djie, Y. Wang, C. E. Dimas, V. Hongpinyo, Y. H. Ding, and B. S. Ooi, "Wavelength tuning and emission width widening of ultrabroad quantum dash interband laser," Appl. Phys. Lett., vol. 93, no. 11, pp. 111101-1-111101-3, 2008.

[24] K. Y. Liou, A. G. Dentai, E. C. Burrows, C. H. Joyner, C. A. Bums, and G. Raybon, "Single-quantum-well strained-layer InGaAsInGaAsP lasers for the wavelength range from 1.43 to $1.55 \mu \mathrm{m}$," IEEE Photon. Technol. Lett., vol. 3, no. 4, pp. 311-313, Apr. 1991.

[25] M. J. Hamp, D. T. Cassidy, B. J. Robinson, Q. C. Zhao, D. A. Thompson, and M. Davies, "Effect of barrier height on the uneven carrier distribution in asymmetric multiple-quantum-well InGaAsP lasers," IEEE Photon. Technol. Lett., vol. 10, no. 10, pp. 1380-1382, Oct. 1998. 


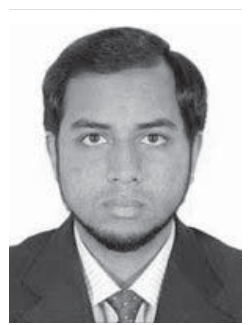

Mohammed Zahed Mustafa Khan received the B.E. degree in electronics and communication from Osmania University, Hyderabad, India, in 2001, and the M.S. degree in electrical engineering from the King Fahd University of Petroleum and Minerals (KFUPM), Dhahran, Saudi Arabia, in 2004. He is currently pursuing the Ph.D. degree in photonics and electromagnetics with the King Abdullah University of Science and Technology, Thuwal, Saudi Arabia, focusing on the theoretical and experimental investigation of quantum-dash lasers.

He was a Lecturer at KFUPM from 2004 to 2009. He is currently with the Photonics Laboratories, King Abdullah University of Science and Technology.

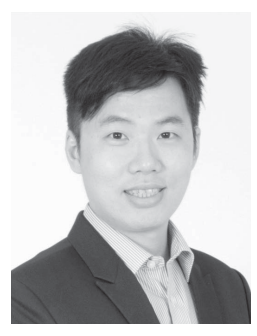

Tien Khee Ng (M'10) received the M.E. and Ph.D. degrees in electrical and electronics engineering from Nanyang Technological University (NTU), Singapore, in 2001 and 2005, respectively.

He joined Tinggi Technologies, a GaN-based highbrightness blue LED start-up company, Singapore, in 2004, as an Engineer, and later as a Technical Staff Member and a Thin Film Process Manager. In 2007, he was a Research Fellow at NTU, where he researched molecular beam epitaxy grown GaAs nanowires and dilute-nitride solar cells using SiGeand/or Si-based heterogeneous substrates. He is currently a Research Scientist with the King Abdullah University of Science and Technology, Thuwal, Saudi Arabia, where he is investigating broad-linewidth arsenic-based and large bandgap nitride-based light sources, as well as the integration of silicon-based optical elements with III-V-based broadband light sources.

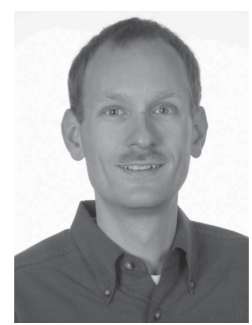

Udo Schwingenschlögl received the M.Sc. and $\mathrm{Ph} . \mathrm{D}$. degrees in physics from the University of Augsburg, Augsburg, Germany, in 2001 and 2004, respectively.

He joined the King Abdullah University of Science and Technology, Thuwal, Saudi Arabia, in 2008, as an Assistant Professor. His current research interests include the electronic and structural properties of nanostructured systems, including surfaces and interfaces.

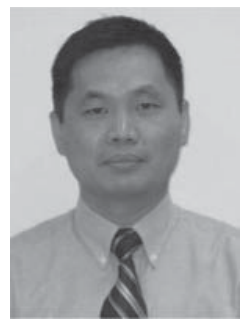

Boon S. Ooi (M'95-SM'03) received the B.E. and $\mathrm{Ph} . \mathrm{D}$. degrees in electronics and electrical engineering from the University of Glasgow, Glasgow, U.K., in 1992 and 1994, respectively.

He was an Assistant Professor with Nanyang Technological University (NTU), Singapore, from 1996 to 2000. From 2003 to 2009, he was an Associate Professor with Lehigh University, Bethlehem, PA. $\mathrm{He}$ is currently a Professor of electrical engineering with the King Abdullah University of Science and Technology, Thuwal, Saudi Arabia. His current research interests include development of semiconductor photonics-integrated circuits and quantum-dot and quantum-dash laser devices.

Prof. Ooi is a fellow of International Society of Optical Engineering and the Institute of Physics, U.K. 